\section{Magnitude do aborto inseguro em Pernambuco, Brasil, 1996 a 2006}

\author{
Unsafe abortion in Pernambuco State, \\ Brazil, 1996-2006
}

\author{
1 Faculdade de Ciências \\ Médicas, Universidade de \\ Pernambuco, Recife, Brasil. \\ 2 Instituto de Medicina \\ Integral Prof. Fernando \\ Figueira, Recife, Brasil. \\ Correspondência \\ F. M. B. Mello \\ Faculdade de Ciências \\ Médicas, Universidade de \\ Pernambuco. \\ Rua Mário de Andrade 446, \\ Caruaru, PE 55026-230, Brasil. \\ fernandambm@yahoo.com.br
}

\section{Abstract}

This study estimates the total number of unsafe abortions and the ratio of unsafe abortions to live births in the State of Pernambuco, Brazil, and in the State's micro-regions (GERES) from 1996 to 2006. A descriptive, ecological, time trend study was performed on the ratio of unsafe abortions to live births. The number of hospital admissions from complications of abortions was obtained from the Hospital Information System (SIH/SUS). The Alan Guttmacher Institute methodology was used to estimate the number of unsafe abortions. The majority of admissions and estimated unsafe abortions occurred in the 1st and 4th GERES. As for the ratio of unsafe abortions to live births, there was an upward trend in the 2nd, 4th, 7th, 9th, 10th, and 11th GERES and a decrease in the $1 s t$, 3rd, 5th, 6th, and 8th GERES, but the trends were only statistically significant in the 1st,5th, 10th, and 11th GERES. In conclusion, Pernambuco showed a high abortion-related hospitalization rate from 1996 to 2007. The estimated number of unsafe abortions was high, with an average of 56,457 per year, 4,705 per month, or 157 per day.

Abortion; Hospitalization; Regression Analysis
Fernanda Maria Bezerra de Mello 1 Jailson Lopes de Sousa 1 José Natal Figueroa 2

\section{Introdução}

No Brasil, o aborto induzido, também chamado de aborto inseguro, é considerado ilegal, uma vez que o Código Penal o inclui entre os crimes contra a vida, exceto em casos de estupro (aborto sentimental) e riscos à vida da mulher grávida (aborto terapêutico) 1. Apesar dessa restrição prevista por lei, a ilegalidade do aborto não tem impedido que ele ocorra indiscriminadamente entre as diferentes classes sociais no Brasil. Mas, certamente, o fato de haver ou não complicações pós-aborto é um evento sócio e economicamente dependente $2,3,4,5$.

Esta relação se deve ao fato de que, enquanto mulheres de classes sociais mais privilegiadas recorrem ao aborto em clínicas privadas com procedimentos seguros, mulheres pertencentes a classes sociais menos favorecidas são expostas a procedimentos inseguros, na maioria das vezes, realizados por profissionais não especializados, com métodos rudimentares e condições de baixa higiene, representado importante causa de morbimortalidade em mulheres 2,3,6,7.

Por outro lado, o aborto considerado seguro é aquele que é realizado nas situações previstas por lei, possibilitando às mulheres o atendimento necessário por parte dos serviços de saúde, os quais, devidamente estruturados, devem oferecer a elas assistência psicossocial no momento da decisão. Além disso, deve garantir a qualidade 
da atenção à saúde necessária para o atendimento e acompanhamento do evento em si 8 .

Desde o fim dos anos 80 e início dos anos 90, em nosso país, vem se observando uma elevação no número de abortos legais ocorridos em hospitais da rede de saúde. Isso configura um assunto de grande interesse à saúde pública, principalmente porque esse número vem aumentando significativamente nos últimos 10 anos 9 .

Assim, o abortamento representa um grave problema de saúde pública e justiça social, principalmente em países em desenvolvimento, onde há uma complexa cadeia de aspectos econômicos, legais, sociais e psicológicos 10 . Pode-se dizer ainda que as poucas evidências científicas sobre seus condicionantes e determinantes demonstram que a ilegalidade, além de trazer conseqüências para a saúde das mulheres, não coíbe a prática e mantém a desigualdade social no que se refere ao aborto $4,11,12$.

Em face do exposto, é possível perceber que estudos que se propõem a aprofundar a temática do aborto inseguro são justificados por várias razões.

Primeiro, por causa da complexidade de se obterem informações estatísticas diretas sobre a magnitude do aborto inseguro em um país que criminaliza o aborto, exceto em duas circunstâncias previstas no Código Penal.

Segundo, o conhecimento sobre a magnitude do aborto inseguro poderá contribuir significativamente para a construção de políticas públicas que promovam a discussão, prevenção e atenção integral e humanizada às mulheres em situação de abortamento.

Terceiro, o aborto inseguro é uma questão relevante na saúde pública, em que pesquisas que aprofundem a questão podem auxiliar na redução da morbimortalidade materna, já que ele se configura como uma de suas principais causas.

Por fim, a maior visibilidade da questão do aborto inseguro contribui para subsidiar a discussão sobre a sua descriminalização e diminuição de seu estigma, estimulando os profissionais, independentemente de seus preceitos morais e religiosos, a preservarem postura ética, garantindo o respeito aos direitos humanos, sexuais e reprodutivos das mulheres.

Dessa forma, este estudo teve o objetivo de estimar a magnitude e a razão de abortos inseguros segundo os nascidos vivos ocorridos no Estado de Pernambuco e nas suas microrregionais de saúde, no período de 1996 a 2006.

\section{Procedimentos metodológicos}

Realizou-se estudo descritivo, do tipo ecológico de tendência temporal, sendo realizada análise espaço-temporal da razão de abortos inseguros por nascidos vivos no Estado de Pernambuco e nas suas microrregionais de saúde, no período de 1996 a 2006.

A análise do recorte territorial baseou-se no Plano Diretor de Regionalização do Estado de Pernambuco, no qual se propõe uma divisão político-administrativa do território em $11 \mathrm{mi}$ crorregiões de saúde, também conhecidas como Gerências Regionais de Saúde (GERES). Estas englobam os 184 municípios do estado e o distrito estadual de Fernando de Noronha, assim distribuídos: GERES I - Região Metropolitana do Recife (18 municípios e 1 distrito estadual); II - Zona da Mata Norte (31 municípios); III Zona da Mata Sul (21 municípios); IV - Agreste Central (32 municípios); V - Agreste Meridional (21 municípios); VI - Sertão do Moxotó (14 municípios); VII - Sertão Central (07 municípios); VIII - Sertão do São Francisco (07 municípios); IX - Sertão do Araripe (11 municípios); X - Sertão do Pajeú (12 municípios) e XI - Sertão de Itaparica (10 municípios).

A escolha do período para análise se deu porque 1996 foi o primeiro ano da décima revisão da Classificação Internacional de Doenças (CID-10) e 2006 foi o último ano pesquisado no banco de dados do Sistema de Informações sobre Nascidos Vivos (SINASC), disponibilizado pela Secretaria Estadual de Saúde de Pernambuco (SES/PE), no momento da coleta de dados para esta pesquisa.

Tratou-se de um estudo com dados secundários, utilizando como fonte o número de internações hospitalares de mulheres por consequência de abortamento (capítulo 15 da CID-10, códigos O00, O02, O03, O04, O06) e o número de nascidos vivos do estado. Estes dados foram obtidos, respectivamente, no Sistema de Informação Hospitalar (SIH/SUS), do Departamento de Informática do SUS (DATASUS), e no SINASC, disponibilizado pelo Núcleo de Epidemiologia da SES/PE.

Os dados de internação hospitalar por conseqüência de aborto subsidiaram a construção das estimativas de aborto inseguro nas regiões do estado. Para isso, utilizou-se a metodologia empregada pelo Instituto Alan Guttmacher 13, que leva em consideração para o cálculo os seguintes pontos: $20 \%$ das mulheres que sofreram aborto tiveram de ser hospitalizadas em conseqüência de suas complicações ( 1 internação para cada 5 abortos); $12,5 \%$ foi considerado como estimativa do sub-registro (abortos realizados fora do SUS); foi descontada uma proporção de $25 \%$ de abortos por causas espontâneas. 
Estas considerações se apresentam na seguinte fórmula:

$\mathrm{N}_{\mathrm{AI}}=(5)(1.125)(0.75) \mathrm{N}_{\mathrm{IH}}$

(Equação 1),

em que $\mathrm{N}_{\mathrm{Al}}$ é o número de abortos inseguros e $\mathrm{N}_{\mathrm{IH}}$ é o número de internações hospitalares por abortamento.

Com a estimativa de abortos inseguros já estabelecida, segundo o ano e região analisados, obteve-se a razão de abortos inseguros segundo o número de nascidos vivos, multiplicando-se por 100, de acordo com a fórmula abaixo:

$\mathrm{R}_{\mathrm{AI}}=100 \frac{\mathrm{N}_{\mathrm{AI}}}{\mathrm{N}_{\mathrm{NV}}}$

(Equação 2),

em que $\mathrm{R}_{\mathrm{AI}}$ é a razão de abortos inseguros por nascidos vivos e $\mathrm{N}_{\mathrm{NV}}$ é o número de nascidos vivos.

As razões do aborto inseguro por nascidos vivos forneceram as taxas de variação média anual no período estudado por meio da regressão linear (Equação da Reta), a qual possibilitou a comparação no tempo entre os valores estimados (observados) e os valores ajustados (esperados) pela referida regressão.

O presente estudo foi submetido ao Comitê de Ética da Universidade de Pernambuco, tendo sido aprovado por seguir todas as normas da $D e$ claração de Helsinki e da Resolução $n^{\circ}$. 196/1996 do Conselho Nacional de Saúde.

\section{Resultados}

Foram analisadas as 147.205 internações hospitalares relacionadas às causas de abortos registrados no SIH/SUS no Estado de Pernambuco, no período de 1996 a 2006, resultando nas seguintes médias: 13.382,3 internações hospitalares por ano; 1.115,2, por mês; 37,2, por dia.

Empregando-se os valores dessas internações hospitalares na Equação 1, foi possível estimar o número de abortos inseguros segundo ano e microrregiões de Pernambuco para o período analisado. Analisando o estado como um todo, estimou-se, para toda a série analisada, um total de 621.022 abortos inseguros, chegando a uma média de 56.457 por ano, 4.705 por mês e 157 por dia (Tabela 1). Considerando-se que, nesse mesmo período, houve uma média de 156.202,5 nascidos vivos no estado por ano, a estimativa da média anual de abortos inseguros representaria $36,1 \%$ dos nascidos vivos de Pernambuco.

A maioria dos casos de internações hospitalares, bem como as estimativas de aborto inseguro, ocorreu nas GERES I e IV, alcançando juntas, no acumulado do período, um total de $63,8 \%$ dos casos. É importante salientar a baixa participação das GERESVII, X e XI, sendo responsáveis por apenas 5,7\% dos casos de internações hospitalares e estimativas de aborto inseguro acumulados em todo o período (Tabela 1).

Ao se observarem os extremos da série histórica, 1996 e 2006, pode-se constatar uma redução de 7,7\% no número estimado de abortos inseguros em Pernambuco. O mesmo comportamento é heterogêneo entre as regiões do estado, havendo redução apenas na região I (-26,6\%). Com comportamento oposto, estão as GERES II $(+2,5 \%)$, III (+31\%), IV (+7,6\%), V (+111,5\%), VI $(+2,8 \%)$, VII (+66,3\%), VIII (+0,4\%), IX (+2,4\%), X (+138,8\%) e XI $(+47,4 \%)$. As regiões V e X chamam a atenção por terem mais que dobrado os casos estimados de abortos inseguros (Tabela 1).

Utilizando-se a Equação 2, foi calculada a razão de abortos inseguros por nascidos vivos no período de 1996 a 2006. Em Pernambuco, quando analisamos a tendência, observamos um comportamento bastante variável entre os anos, pois houve momentos de elevação e outros de redução dessa razão, quando comparamos os valores observados com os valores esperados (ajustados) da equação da reta. Fazendo a mesma análise por GERES, observamos variabilidade semelhante à encontrada para o estado (Tabelas 2 e 3).

As taxas de variação média anual da razão observada e ajustada da estimativa de abortos inseguros por nascidos vivos, no período de 1996 a 2006, revelam tendência estatisticamente significante de diminuição anual na GERES I $(-0,911)$ e de aumento na X $(+2,153)$ e XI $(+1,499)$. Nas demais GERES, assim como no Estado de Pernambuco, não houve variação estatisticamente significativa da média anual (Tabela 3).

\section{Discussão}

Ao falar sobre aborto inseguro, é preciso antes de tudo relatar a complexidade de se mensurar sua ocorrência, pois o aborto, salvo nos casos previstos por lei, é crime no nosso país, o que dificulta sua notificação. Outro fator concorrente no conhecimento da sua magnitude é que, no Brasil, os dados obtidos sobre abortamentos são baseados fundamentalmente no número de registros de internações hospitalares por suas complicações, porém esses dados apresentam uma grande quantidade de sub-registros, já que o SIH/SUS só tem abrangência para os casos com acesso aos serviços públicos de saúde, excluindo as pessoas que não tiveram acesso ao SUS, as que tiveram e não foram internadas e as internadas na rede privada de saúde 14,15 . 
Estimativa de abortos inseguros * segundo ano e Gerências Regionais de Saúde (GERES). Pernambuco, Brasil, 1996-2006.

\begin{tabular}{|c|c|c|c|c|c|c|c|c|c|c|c|c|c|}
\hline \multicolumn{2}{|c|}{ GERES } & \multirow{2}{*}{$\begin{array}{c}1996 \\
33.015,9\end{array}$} & \multirow{2}{*}{$\begin{array}{c}1997 \\
34.846,9\end{array}$} & \multirow{2}{*}{$\begin{array}{c}1998 \\
31.442,3\end{array}$} & \multirow{2}{*}{$\begin{array}{c}1999 \\
30.864,4\end{array}$} & \multirow{2}{*}{$\begin{array}{c}2000 \\
31.421,3\end{array}$} & \multirow{2}{*}{$\begin{array}{c}2001 \\
30.315,9\end{array}$} & \multirow{2}{*}{$\begin{array}{c}2002 \\
29.788,6\end{array}$} & \multirow{2}{*}{$\begin{array}{c}2003 \\
25.084,7\end{array}$} & \multirow{2}{*}{$\begin{array}{c}2004 \\
27.772,0\end{array}$} & \multirow{2}{*}{$\begin{array}{c}2005 \\
28.438,6\end{array}$} & \multirow{2}{*}{$\begin{array}{c}2006 \\
24.236,7\end{array}$} & \multirow{2}{*}{$\begin{array}{c}\text { Total } \\
327.227,3\end{array}$} \\
\hline I & $\mathrm{n}$ & & & & & & & & & & & & \\
\hline & $\%$ & 58,8 & 56,0 & 52,8 & 56,9 & 55,4 & 52,5 & 49,7 & 50,2 & 50,7 & 49,3 & 46,7 & 52,7 \\
\hline \multirow[t]{2}{*}{ II } & $\mathrm{n}$ & $3.720,9$ & $3.754,7$ & $3.868,6$ & $3.813,8$ & $3.720,9$ & $3.640,8$ & $3.771,6$ & $3.332,8$ & $3.645,0$ & $3.902,3$ & $3.813,8$ & $40.985,2$ \\
\hline & $\%$ & 6,6 & 6,0 & 6,5 & 7,0 & 6,6 & 6,3 & 6,3 & 6,7 & 6,7 & 6,8 & 7,4 & 6,6 \\
\hline \multirow[t]{2}{*}{ III } & $n$ & $2.134,7$ & $3.172,5$ & $2.792,8$ & $2.438,4$ & $2.657,8$ & $2.421,6$ & $3.320,2$ & $2.556,6$ & $2.151,6$ & $2.421,6$ & $2.797,0$ & $28.864,7$ \\
\hline & $\%$ & 3,8 & 5,1 & 4,7 & 4,5 & 4,7 & 4,2 & 5,5 & 5,1 & 3,9 & 4,2 & 5,4 & 4,6 \\
\hline \multirow[t]{2}{*}{ IV } & $\mathrm{n}$ & $6.412,5$ & $7.399,7$ & $6.640,3$ & $3.678,8$ & $4.282,0$ & $6.231,1$ & $6.986,3$ & $5.636,3$ & $7.066,4$ & $7.648,6$ & $6.897,7$ & $68.879,7$ \\
\hline & $\%$ & 11,4 & 11,9 & 11,2 & 6,8 & 7,6 & 10,8 & 11,7 & 11,3 & 12,9 & 13,3 & 13,3 & 11,1 \\
\hline \multirow[t]{2}{*}{ V } & $\mathrm{n}$ & $1.316,3$ & $1.417,5$ & $3.961,4$ & $3.358,1$ & $3.484,7$ & $3.235,8$ & $3.720,9$ & $3.045,9$ & $3.071,3$ & $3.012,2$ & $2.784,4$ & $32.408,5$ \\
\hline & $\%$ & 2,3 & 2,3 & 6,7 & 6,2 & 6,1 & 5,6 & 6,2 & 6,1 & 5,6 & 5,2 & 5,4 & 5,2 \\
\hline \multirow[t]{2}{*}{ VI } & $\mathrm{n}$ & $1.826,7$ & $2.421,6$ & $1.776,1$ & $1.957,5$ & $2.151,6$ & $2.269,7$ & $2.413,1$ & $1.894,2$ & $2.240,2$ & $2.075,6$ & $1.877,3$ & $22.903,6$ \\
\hline & $\%$ & 3,3 & 3,9 & 3,0 & 3,6 & 3,8 & 3,9 & 4,0 & 3,8 & 4,1 & 3,6 & 3,6 & 3,7 \\
\hline \multirow[t]{2}{*}{ VII } & $n$ & 725,6 & $1.489,2$ & $1.324,7$ & $1.147,5$ & $1.046,3$ & $1.084,2$ & $1.042,0$ & 953,4 & 983,0 & $1.177,0$ & $1.206,6$ & $12.179,5$ \\
\hline & $\%$ & 1,3 & 2,4 & 2,2 & 2,1 & 1,8 & 1,9 & 1,7 & 1,9 & 1,8 & 2,0 & 2,3 & 2,0 \\
\hline \multirow[t]{2}{*}{ VIII } & $\mathrm{n}$ & $3.438,3$ & $3.159,8$ & $3.640,8$ & $3.737,8$ & $3.860,2$ & $3.324,4$ & $3.708,3$ & $3.311,7$ & $3.404,5$ & $4.130,2$ & $3.450,9$ & $39.166,9$ \\
\hline & $\%$ & 6,1 & 5,1 & 6,1 & 6,9 & 6,8 & 5,8 & 6,2 & 6,6 & 6,2 & 7,2 & 6,7 & 6,3 \\
\hline \multirow[t]{2}{*}{ IX } & $n$ & $2.138,9$ & $2.632,5$ & $2.438,4$ & $1.700,2$ & $1.949,1$ & $2.944,7$ & $2.830,8$ & $2.050,3$ & $1.970,2$ & $2.316,1$ & $2.189,5$ & $25.160,6$ \\
\hline & $\%$ & 3,8 & 4,2 & 4,1 & 3,1 & 3,4 & 5,1 & 4,7 & 4,1 & 3,6 & 4,0 & 4,2 & 4,1 \\
\hline \multirow[t]{2}{*}{ X } & $n$ & 489,4 & 649,7 & 780,5 & 675,0 & 814,2 & 898,6 & $1.092,7$ & 793,1 & $1.067,3$ & $1.088,4$ & $1.168,6$ & $9.517,5$ \\
\hline & $\%$ & 0,9 & 1,0 & 1,3 & 1,2 & 1,4 & 1,6 & 1,8 & 1,6 & 2,0 & 1,9 & 2,3 & 1,5 \\
\hline \multirow[t]{2}{*}{$X I$} & $\mathrm{n}$ & 970,3 & $1.290,9$ & 873,3 & 877,5 & $1.324,7$ & $1.434,4$ & $1.316,3$ & $1.354,2$ & $1.362,7$ & $1.493,4$ & $1.430,2$ & $13.727,8$ \\
\hline & $\%$ & 1,7 & 2,1 & 1,5 & 1,6 & 2,3 & 2,5 & 2,2 & 2,7 & 2,5 & 2,6 & 2,8 & 2,2 \\
\hline \multirow[t]{2}{*}{ Total } & $n$ & $56.190,0$ & $62.235,0$ & $59.539,0$ & $54.249,0$ & $56.713,0$ & $57.801,0$ & $59.991,0$ & $50.013,0$ & $54.734,0$ & $57.704,0$ & $51.853,0$ & $621.022,0$ \\
\hline & $\%$ & 100,0 & 100,0 & 100,0 & 100,0 & 100,0 & 100,0 & 100,0 & 100,0 & 100,0 & 100,0 & 100,0 & 100,0 \\
\hline
\end{tabular}

Fonte: Sistema de Informação Hospitalar (SIH/SUS).

* $\mathrm{N}_{\mathrm{Al}}=(5)(1.125)(0.75) \mathrm{N}_{\mathrm{HH}}$, em que $\mathrm{N}_{\mathrm{Al}}$ : número de abortos inseguros; $\mathrm{N}_{\mathrm{HH}}$ : número de internações hospitalares por abortamento.

Tabela 2

Razão de abortos inseguros por 100 nascidos vivos *, segundo ano e Gerências Regionais de Saúde (GERES). Pernambuco, Brasil, 1996-2006.

\begin{tabular}{cccccccccccccc}
\hline Ano & & & & & & \multicolumn{3}{c}{ GERES } & & & & & \\
& & I & II & III & IV & V & VI & VII & VIII & IX & X & XI & Total \\
\hline 1996 & 52,71 & 22,41 & 22,28 & 29,74 & 11,62 & 24,20 & 22,71 & 45,12 & 27,43 & 12,99 & 20,34 & 291,54 \\
1997 & 54,64 & 29,92 & 26,93 & 35,36 & 12,65 & 33,43 & 45,27 & 42,82 & 41,73 & 18,22 & 26,88 & 367,83 \\
1998 & 46,13 & 27,11 & 23,30 & 29,40 & 35,73 & 27,01 & 42,69 & 46,36 & 32,16 & 22,98 & 19,32 & 352,19 \\
1999 & 43,63 & 26,74 & 19,15 & 15,79 & 31,24 & 26,85 & 38,77 & 42,60 & 23,98 & 24,18 & 22,83 & 315,76 \\
2000 & 45,73 & 24,51 & 23,31 & 18,97 & 33,53 & 27,76 & 33,09 & 44,08 & 24,10 & 24,41 & 32,79 & 332,27 \\
2001 & 45,31 & 24,90 & 18,97 & 26,97 & 30,42 & 27,66 & 35,84 & 37,07 & 36,51 & 27,31 & 31,86 & 342,81 \\
2002 & 47,46 & 26,90 & 28,31 & 31,23 & 35,64 & 29,02 & 34,22 & 43,40 & 38,65 & 33,92 & 30,78 & 379,53 \\
2003 & 40,39 & 23,80 & 22,60 & 26,97 & 29,44 & 24,06 & 32,66 & 41,13 & 30,46 & 24,97 & 31,85 & 328,33 \\
2004 & 45,50 & 25,83 & 19,14 & 34,06 & 31,63 & 29,28 & 34,43 & 42,16 & 28,29 & 34,14 & 33,18 & 357,64 \\
2005 & 46,31 & 27,25 & 21,41 & 35,66 & 30,22 & 26,66 & 40,46 & 48,45 & 32,13 & 33,90 & 35,22 & 377,67 \\
2006 & 40,66 & 28,54 & 25,76 & 33,76 & 28,20 & 25,33 & 44,18 & 41,39 & 33,93 & 38,89 & 35,10 & 375,76 \\
\hline
\end{tabular}

Fonte: Sistema de Informação Hospitalar (SIH/SUS) e Sistema de Informações sobre Nascidos Vivos (SINASC), disponibilizado pela Secretaria Estadual de Saúde de Pernambuco (SES/PE).

${ }^{*} \mathrm{R}_{\mathrm{Al}}=100 \frac{\mathrm{N}_{\mathrm{AI}}}{\mathrm{N}_{\mathrm{NV}}}$, em que $\mathrm{R}_{\mathrm{AI}}$ : razão de abortos inseguros por nascidos vivos; $\mathrm{N}_{\mathrm{NV}}$ : número de nascidos vivos. 
Componentes da Equação da Reta (regressão linear), valor de p e coeficiente de determinação da razão de abortos inseguros por 100 nascidos vivos, segundo Gerências Regionais de Saúde (GERES). Pernambuco, Brasil, 1996-2006.

\begin{tabular}{|c|c|c|c|c|}
\hline GERES & $\begin{array}{c}\text { Constante } \\
\qquad(\alpha)\end{array}$ & $\begin{array}{c}\text { Taxa de variação média } \\
\text { anual }(\beta)\end{array}$ & $\begin{array}{c}\text { Valor de } \\
p\left(H_{0}: \beta=0\right)\end{array}$ & $\begin{array}{c}\text { Coeficiente de } \\
\left.\text { determinação ( } \mathbf{R}^{2}\right)\end{array}$ \\
\hline I & 50,8 & $-0,91$ & 0,017 & 48,6 \\
\hline II & 25,6 & 0,11 & 0,669 & 3,1 \\
\hline III & 23,1 & $-0,05$ & 0,865 & 0,3 \\
\hline IV & 25,7 & 0,64 & 0,270 & 10,5 \\
\hline V & 35,6 & $-0,63$ & 0,057 & 42,6 \\
\hline $\mathrm{VI}$ & 28,2 & $-0,17$ & 0,602 & 4,7 \\
\hline VII & 34,4 & 0,47 & 0,575 & 5,9 \\
\hline VIII & 43,7 & $-0,11$ & 0,688 & 1,5 \\
\hline IX & 31,3 & 0,09 & 0,864 & 0,3 \\
\hline$x$ & 16,1 & 2,153 & $<0,001$ & 85,5 \\
\hline$X I$ & 21,6 & 1,499 & $<0,001$ & 73,0 \\
\hline Total & 36,7 & $-0,10$ & 0,655 & 2,0 \\
\hline
\end{tabular}

A metodologia do Instituto Alan Guttmacher 13 , utilizada neste estudo para estimar o número de abortos inseguros no estado, leva em consideração percentuais propostos de sub-registro e de abortos espontâneos. Mostrase como um parâmetro de fácil cálculo e bem oportuno para dimensionar o aborto inseguro em sociedades que ainda o criminalizam, já que apresenta proposta metodológica interessante para correção destes sub-registros.

A análise dos resultados obtidos aponta para um alto número de internações hospitalares em conseqüência do aborto em toda a extensão do estado. Conforme alguns autores 14,16, esse número elevado deve-se ao fato de o aborto ser considerado crime pelo Código Penal brasileiro, com exceção apenas para os casos de estupro e risco iminente de vida da mãe, levando as mulheres a procurarem serviços clandestinos e muitas vezes não seguros para essa prática.

A literatura já aponta que, mesmo havendo a ilegalidade do aborto provocado em muitos países, ele continua ocorrendo indiscriminadamente entre as diferentes classes sociais. Mas, certamente, o fato de ter ou não complicação pós-aborto tem relação com as condições socioeconômicas e culturais das mulheres 3,14,16.

No que se refere à relação socioeconômica, é interessante ressaltar que mulheres de classes sociais mais privilegiadas têm acesso a clínicas privadas com procedimentos mais seguros, diferentemente de mulheres pertencentes a classes sociais menos favorecidas, as quais são expostas a procedimentos inseguros e, na maioria das vezes, realizados por profissionais desqualificados, com métodos rudimentares e condições precárias $3,11,14,16$.

No caso de Pernambuco, há média mensal de 4.705 abortos inseguros e média diária de 157 . Esses resultados estão coerentes com estimativas para o Brasil, com 87.854 abortos por mês e 2.929 por dia, embora este estudo para o país não tenha sofrido nenhum tipo de correção para a questão da subnotificação 17 .

Os dados obtidos mostram que a distribuição da estimativa do aborto inseguro ocorreu de maneira desigual ao longo de todo o estado. Há regiões com comportamentos de variação média anual de crescimento na série e outras de redução, ainda que três tenham tido resultados estatisticamente significantes (GERES I, X e XI). Essas três regiões também apresentaram os maiores coeficientes de determinação ( $\left.\mathrm{R}^{2}\right)$, ou seja, são as que apresentaram as maiores linearidades do modelo. Isso se deve, provavelmente, à própria heterogeneidade entre as regiões, pois o aborto apresenta um peso maior nas taxas de morbimortalidade materna principalmente em populações socialmente vulneráveis e em regiões com menor acesso a serviços de saúde resolutivos e de qualidade 16 .

A média anual da estimativa de aborto em Pernambuco alcançou $36,1 \%$ da média de nascidos vivos para o período estudado, mostrando que uma elevada proporção de mulheres recorreu à prática por diversas questões. Esse percentual para o estado ficou acima do encontrado para o Brasil no ano de 2005, quando o país alcançou uma proporção de $35 \% 17$. 
Não obstante verificar-se redução da estimativa de abortos inseguros em Pernambuco entre os anos extremos da série analisada, 1996 e 2006, a literatura ${ }^{9}$ aponta que, no Brasil, a partir do fim dos anos 80 e início dos anos 90, vem se observando uma elevação no número de abortos ocorridos em hospitais da rede pública. Esse aumento também se deu, provavelmente, pelas recentes aberturas na nossa legislação, já que, a partir de 1998, alguns juristas passaram a considerar certas malformações congênitas como condições favoráveis para o aborto legal, o que ampliou o leque de situações neste caso.

O maior acesso à rede do SUS e a ampliação da jurisprudência em relação ao código civil brasileiro podem ser fatores que ajudam a explicar a redução de abortos inseguros no Estado de Pernambuco, pois a maior disponibilidade do aborto legal sob condições favoráveis reduz a exposição à sua prática clandestina. Como exemplo dessa questão, hoje, mulheres que sofreram violência sexual já podem recorrer a serviços públicos de saúde para realizarem a interrupção da gestação de forma assistida e segura.

Ao mostrar a realidade de Pernambuco, podemos mencionar também a relevância do tema em nível mundial: aproximadamente 26 milhões de abortos foram realizados legalmente e $20 \mathrm{mi}-$ lhões, ilegalmente, no ano de 1995. Assim, a literatura mostra que a restrição legal não garante uma baixa taxa de abortamentos; ao contrário, as taxas mais altas de abortos são observados naqueles países onde a prática é restringida por lei, sendo a maioria deles realizados de forma insegura 17.

Constata-se que, em países com leis restritivas, o aborto inseguro constitui-se uma importante causa de mortalidade e de morbidade materna. Para alguns autores, quando o aborto induzido é realizado precocemente por médicos experientes e com as condições mínimas neces- sárias, seu índice de segurança será elevado. Alguns estudos acrescentam, ainda, que, em países onde os abortamentos são praticados de forma legal e segura, em geral, a morte materna é evento raro e, quando ocorre, está relacionada a complicações anestésicas 11,14.

Em 2006, a Organização Mundial da Saúde (OMS) estimou que, nos países em desenvolvimento, cerca de $15 \%$ das mortalidades maternas são decorrentes de complicações de aborto, podendo atingir cerca de $50 \%$ dessa mortalidade dependendo do local ${ }^{3}$.

Em Cuba, a partir da legalização do aborto, a mortalidade materna declinou de 120 por 100 mil nascidos vivos para 7 por 100 mil nascidos vivos, em estudo publicado em 2002. Isso significa que legalizar o aborto, realizando-o em adequadas condições sanitárias e por profissional habilitado, possibilitou reduzir drasticamente a morte materna 16 .

Em síntese, a alta estimativa de ocorrência diária apontada por este estudo configura o aborto inseguro como problema de saúde pública em Pernambuco, trazendo implicações para o próprio sistema de saúde, com elevadas taxas de morbimortalidade em virtude de complicações pela sua realização insegura e clandestina. Mas esse fato leva, principalmente, a implicações sociais, psicológicas e de qualidade de vida para essas mulheres e suas famílias.

O enfrentamento desse problema perpassa por questões profundas de justiça social, ética, legislação civil, cidadania e direitos sexuais e reprodutivos. Políticas públicas só serão efetivas quando pautadas em maior conhecimento sobre a cadeia de causalidade do aborto inseguro, o que pode ser alcançado a partir de um maior número de estudos com metodologia específica para o entendimento dos seus determinantes e condicionantes. 


\section{Resumo}

Este estudo estima a magnitude e a razão de abortos inseguros segundo os nascidos vivos, em Pernambuco, Brasil, e nas microrregionais de saúde do estado, entre 1996 e 2006. Realizou-se um estudo descritivo, ecológico, de tendência temporal, com análise espaço-temporal da razão de abortos inseguros. Obteve-se o número de internações hospitalares de mulheres por complicacões de aborto do Sistema de Informação Hospitalar (SIH/SUS); utilizou-se a metodologia empregada pelo Instituto Alan Guttmacher para estimar o número de abortos inseguros. Verificou-se que a maioria das internações e das estimativas de aborto inseguro ocorreu nas GERES I e IV. Em relação à razão das estimativas de aborto inseguro por nascidos vivos, houve tendência de crescimento nas GERES II, IV, VII, IX, X e XI e redução nas GERES I, III, V, VI e VIII, mas apenas as GERES I, V, X e XI têm relação estatisticamente significante. Conclui-se que Pernambuco apresentou uma alta taxa de internação por aborto entre 1996 e 2007. A estimativa de abortos inseguros se apresenta elevada, com de média de 56.457 ao ano, 4.705 por mês e 157 por dia.

Aborto; Hospitalização; Análise de Regressão

\section{Colaboradores}

F. M. B. Mello contribuiu na concepção do projeto, coleta de dados, análise e interpretação dos dados e redação do artigo. J. L. Sousa participou da concepção do projeto, análise e interpretação dos dados, além da revisão crítica do artigo. J. N. Figueroa participou da análise estatística, interpretação dos dados e revisão crítica do artigo.

\section{Referências}

1. Diniz D. Quem autoriza o aborto seletivo no Brasil? Médicos, promotores e juízes em cena, Brasil. Physis (Rio J.) 2003; 13:13-34.

2. Brasil. Decreto-lei no. 2.848. Código penal. Diário Oficial da União 1940; $31 \mathrm{dez}$.

3. Olinto MTA, Moreira-Filho DC. Fatores de risco e preditores para o aborto induzido: estudo de base populacional. Cad Saúde Pública 2006; 22:365-75.

4. Diniz D. Aborto e a saúde pública no Brasil. Cad Saúde Pública 2007; 23:1992-3.

5. Nader PRA, Macedo CR, Miranda AE, Maciel ELN. Aspectos sociodemográficos e reprodutivos do abortamento induzido de mulheres internadas em uma maternidade do município de Serra do Espírito Santo. Esc Anna Nery Rev Enferm 2008; 12:699-705.

6. Verardo MT. Aborto: um direito ou um crime? São Paulo: Editora Moderna; 1995.

7. Sedgh G. Induced abortion: estimated rates and trends worldwide. Lancet 2007; 370:1338-45.

8. Rede Mundial de Mujeres por los Derechos Reproductivos. Informe de la campaña contra la mortalidad y morbidad materna. Amsterdã: Drukkerij Quint; 1993.

9. Kac G, Silveira EA, Oliveira LC, Araújo EB. Fatores associados à ocorrência de cesárea e aborto em mulheres selecionadas em um centro de saúde no município do Rio de Janeiro, Brasil. Rev Bras Saúde Matern Infant 2007; 7:271-9.
10. Cook RBB, Tathala M. Saúde reprodutiva e direitos humanos: integrando medicina, ética e direito. Rio de Janeiro: Cepia; 2004.

11. Souza KV, Almeida MRCS, Soares VMN. O perfil da mortalidade materna por aborto no Paraná: 20032005. Esc Anna Nery Rev Enferm 2008; 12:741-9.

12. Faúndes A, Duarte GA, Andalaft Neto J, Olivatto AE, Simoneti RM. Conhecimento, opinião e conduta de ginecologistas e obstetras brasileiros sobre o aborto induzido. Rev Bras Ginecol Obstet 2004; 26:89-96.

13. Alan Guttmacher Institute. Clandestine abortion: a Latin American reality. New York: Alan Guttmacher Institute; 1994.

14. Souza AI, Aquino MMA, Cecatti JG, Silva JLP. Epidemiologia do abortamento na adolescência. Rev Bras Ginecol Obstet 1999; 21:161-5.

15. Gomes EC, Menezes RA. Dilemas contemporâneos sobre os limites da vida. Physis (Rio J.) 2008; 18: 77-103.

16. Fusco CLB, Andreone S, Silva RS. Epidemiologia do aborto inseguro em uma população em situação de pobreza Favela Inajar de Souza, São Paulo. Rev Bras Epidemiol 2008; 11:78-88.

17. Silva RS. Padrões de aborto provocado na Grande São Paulo, Brasil. Rev Saúde Pública 1998; 32:7-17.

Recebido em 17/Mai/2010

Versão final reapresentada em 19/Set/2010

Aprovado em 29/Set/2010 